\title{
Medical management of PAD: Expand or consolidate?
}

\author{
Colum R Keohane ${ }^{1}$ MRCSI, Mark Twyford ${ }^{1}$ MRCSI, Evelyn $\underline{\text { Hannon }}{ }^{2}$ MRCPI, Wael Tawfick ${ }^{1}$ MD, Steward R Walsh ${ }^{1}{ }_{F R C S}$
}

In this issue of the Annals, a rapid review of adherence to evidence-based medical treatment ${ }^{1}$ highlights an important and underappreciated aspect of the management of patients with peripheral arterial disease (PAD). It is easy, upon meeting a patient, to prescribe appropriate medication in an outpatient setting and believe that one's job is done; that the patient's risk has been optimised. It is clear from this review, and from previous work, ${ }^{2}$ that this is not the case.

The authors conclude that adherence to evidencebased medical treatment is extremely variable, and that undertreatment is common. The variability in adherence is probably less surprising than how low adherence rates are in most of the studies compiled. For lipidlowering medications, some of the larger and more recent included studies show encouraging adherence rates; however, only 10 of the 41 studies on lipid-lowering drugs report over $75 \%$ adherence. Over half (24/42) of the included studies, including the majority of the larger studies, showed between 50 and $75 \%$ adherence, while over 1 in 6 studies (7/41) reported adherence below $50 \%$. The adherence to antiplatelet medication is more encouraging. While the majority of larger reports again fall between 50 and $75 \%$, a much greater proportion (17/31) report adherence above $75 \%$, and far fewer (3/31) below $50 \%$.

There are often legitimate reasons for non-adherence with or non-prescribing of all of these medications, such as muscle cramps or liver function derangement with statins, or bleeding complications and gastrointestinal (GI) upset with antiplatelets. The use of "any antiplatelet" is more appropriate than singling out aspirin, as this reflects current guidelines and some patients may take clopidogrel given its more favourable side-effect profile regarding GI bleeding. ${ }^{3}$ The lowest adherence rate in the entire review was in a study recording only aspirin use, which is perhaps instructive. This study is an outlier, and had other antiplatelets been included, the result may have been markedly different or at least more in line with the other studies.
Interpretation is more difficult in the case of antidiabetic and antihypertensive medication. The authors are of course limited in the inferences that can be drawn from each study, given the heterogeneity in reporting of both hypertensive patients and hypertensive medication use in individual publications. It is clear however that there is extreme variability in adherence to medication. It is encouraging to see that adherence rates have generally improved over time but it is clear as the authors suggest that improved adherence strategies are needed along with implementation studies to gauge the effect of this. The impact of nurse-led clinics to maximise adherence reported by Sillesen et al. ${ }^{4}$ suggests a way forward in this regard. Anecdotally, in our practice, it is common to find patients have stopped, or their primary care doctor has stopped, a medication outright when dose alteration or a change to another medication in a similar class may have been more appropriate - such as using a different statin or changing antiplatelet.

Numerous recent studies have shown that a variety of medications can help either with symptom control or in reducing adverse cardiovascular events in patients with PAD. When viewed in the context of this ever-expanding repertoire of medications, this latest review forces a separate question to be confronted: in the attempt to medically manage PAD, should the focus be to introduce new and improved treatments into practice? Or should we first aim to maximise adherence to existing guidelines?

Large multicentre randomised trials have shown a modest but significant benefit in patients with PAD, both primarily and after revascularisation, to taking a combination of aspirin and low-dose rivaroxaban. ${ }^{5,6}$ Pentoxyfylline and cilostazol are sometimes prescribed to help in the management of PAD symptoms. A Cochrane review showed uncertain benefit with pentoxyfylline due to poor-quality heterogenous evidence $;$ in another review, cilostazol was considered beneficial in improving walking distance, but with an increase in adverse side effects that were generally mild and treatable. ${ }^{8}$ Neither of these medications are

\footnotetext{
${ }^{1}$ Discipline of Surgery, Lambe Institute for Translational Research, National University of Ireland, Galway, Ireland

${ }^{2}$ Department of Geriatric Medicine, Cork University Hospital, Cork, Ireland

Correspondence: Prof Stewart R Walsh, Discipline of Surgery, Lambe Institute for Translational Research (Floor 2), National University of Ireland, Galway, H91 V4AY, Ireland.

Email: stewartredmond.walsh@nuigalway.ie
} 
recommended in the European guidelines, although cilostazol is recommended by the American College of Cardiology/American Heart Association (ACC/AHA). ${ }^{9}$ Both are frequently prescribed.

These medications all show promise in the management of PAD. One must wonder however what negative effects their addition may have. A previously well claudicant could in a single outpatient visit be commenced on aspirin and a statin, cilostazol and low-dose rivaroxaban. This patient could go from no daily medications to 4 , with more than once daily dosing. A similar patient with diabetes or hypertension, or both, could soon be on 6 or more separate medications with complex dosing schedules. That is to say nothing of the comorbid patient who may already be on multiple medications.

This presents 3 significant challenges. First, more than once daily dosing is a significant risk factor for nonadherence. ${ }^{10,11}$ In both the COMPASS and VOYAGER PAD trials, non-adherence to trial medications was high, with a $16.5 \%$ overall non-adherence ${ }^{6}$ and annualised discontinuation rate of $14 \%$ per year, ${ }^{5}$ respectively. It is reasonable to expect that, outside the more controlled conditions of a randomised trial, real-world adherence rates could be lower still. A large part of this may have been due to the association between multiple daily dosing and non-adherence.

Second, as the authors of this review have correctly identified, polypharmacy is one of a number of patient and physician factors that can contribute to nonadherence. Multiple medications can be a necessary evil in the appropriate management of many conditions, and in younger patients many medications may be manageable. However, in older adults who often have multiple comorbidities, more medications and altered physiology, polypharmacy increases the risk of adverse drug events. ${ }^{12}$ These patients may be prone to prescribing cascades where additional medications are prescribed for new symptoms or ailments without first considering that existing medications may be the cause of those symptoms. This can lead to increasingly complex medication regimens, with each additional medication increasing the risk of side effects, ${ }^{13}$ rates of medication errors $^{13}$ and non-adherence. ${ }^{11}$

Finally, the additional medications that can be used in PAD outside those highlighted in the review, such as cilostazol and rivaroxaban, are not benign. Cilostazol poses a risk of polypharmacy in treating its multiple "easily managed" side effects. The combination of aspirin and rivaroxaban will lead to an increased bleeding risk compared to aspirin alone. While the benefits may outweigh the risks in COMPASS $^{6}$ and VOYAGER PAD, ${ }^{5}$ it is not certain how this plays out in the real world with concurrent use of hypoglycaemic medications and antihypertensives by frail patients who tend to be underrepresented in trials.

As newer treatments emerge and their evidence base grows, their adoption into guidelines and clinical practice must take into account the population in which PAD predominates. Our patients tend to be older, frequently have comorbidities and are all at high risk of polypharmacy. Younger and fitter patients may benefit from an aggressive, multimodal approach to medical management of their PAD. For others, that approach means increased risk of errors, non-adherence, and side effects. It is important that the treatments we prescribe for older adults in particular, align themselves with "what matters most" to that particular person. Current Emergency Cardiac Care (ECC) and ACC/AHA guidelines recommend lifelong aspirin and a statin, as well as management of hypertension and diabetes where indicated, in patients with PAD. Adherence to these medications is already lower than what we would hope, so an important step before considering addition of further medications is to focus on ensuring patients are appropriately prescribed, and compliant with core evidence-based best medical therapy.

\section{REFERENCES}

1. Chan SL, Rajesh R, Tang TY. Evidence-based medical treatment of peripheral arterial disease: A rapid review. Ann Acad Med Singap 2021;50:411-24

2. Flu HC, Tamsma JT, Lindeman JH, et al. A systematic review of implementation of established recommended secondary prevention measures in patients with PAOD. Eur J Vasc Endovasc Surg 2010; 39:70-86.

3. CAPRIE Steering Committee. A randomised, blinded, trial of clopidogrel versus aspirin in patients at risk of ischaemic events (CAPRIE). Lancet 1996;348:1329-39.

4. Sillesen H, Madelung S, Eldrup N, et al. Organising a nurse-driven PAD rehabilitation clinic within the vascular surgical department: what is required and are treatment goals reached-a prospective study? Eur J Vasc Endovasc Surg 2007;33:26-32.

5. Bonaca MP, Bauersachs RM, Anand SS, et al. Rivaroxaban in Peripheral Artery Disease after Revascularization. N Engl J Med 2020;382:1994-2004.

6. Anand SS, Bosch J, Eikelboom JW, et al. Rivaroxaban with or without aspirin in patients with stable peripheral or carotid artery disease: an international, randomised, double-blind, placebocontrolled trial. Lancet 2018;391:219-29.

7. Broderick C, Forster R, Abdel-Hadi M, et al. Pentoxifylline for intermittent claudication. Cochrane Database Syst Rev 2020;10:Cd005262.

8. Bedenis R, Stewart M, Cleanthis M, et al. Cilostazol for intermittent claudication. Cochrane Database Syst Rev 2014;2014:CD003748. 
9. Gerhard-Herman MD, Gornik HL, Barrett C, et al. 2016 AHA/ACC Guideline on the Management of Patients With Lower Extremity Peripheral Artery Disease: Executive Summary: A Report of the American College of Cardiology/American Heart Association Task Force on Clinical Practice Guidelines. Circulation 2017; 135:e686-725.

10. Hughes CM. Medication non-adherence in the elderly: how big is the problem? Drugs Aging 2004;21:793-811.
11. Gellad WF, Grenard JL, Marcum ZA. A systematic review of barriers to medication adherence in the elderly: looking beyond cost and regimen complexity. Am J Geriatr Pharmacother 2011;9:11-23.

12. Davies EA, O'Mahony MS. Adverse drug reactions in special populations - the elderly. Br J Clin Pharmacol 2015;80:796-807.

13. Gandhi TK, Weingart SN, Borus J, et al. Adverse drug events in ambulatory care. N Engl J Med 2003;348:1556-64. 